BNL-113213-2016-JA

File \# 94162

\title{
New figuring model based on surface slope profile for grazing incidence reflective optics
}

\author{
Lin Zhou, Lei Huang, Nathalie Bouet, Konstantine Kaznatcheev, \\ Matthew Vescovi, Yifan Dai, Shengyi Li and Mourad Idir
}

Submitted to the Journal of Synchrotron Radiation

September 2016

Photon Sciences Department

Brookhaven National Laboratory

\author{
U.S. Department of Energy \\ USDOE Office of Science (SC), \\ Basic Energy Sciences (BES) (SC-22)
}

Notice: This manuscript has been authored by employees of Brookhaven Science Associates, LLC under Contract No. DE- SC0012704 with the U.S. Department of Energy. The publisher by accepting the manuscript for publication acknowledges that the United States Government retains a non-exclusive, paid-up, irrevocable, world-wide license to publish or reproduce the published form of this manuscript, or allow others to do so, for United States Government purposes. 


\section{DISCLAIMER}

This report was prepared as an account of work sponsored by an agency of the United States Government. Neither the United States Government nor any agency thereof, nor any of their employees, nor any of their contractors, subcontractors, or their employees, makes any warranty, express or implied, or assumes any legal liability or responsibility for the accuracy, completeness, or any third party's use or the results of such use of any information, apparatus, product, or process disclosed, or represents that its use would not infringe privately owned rights. Reference herein to any specific commercial product, process, or service by trade name, trademark, manufacturer, or otherwise, does not necessarily constitute or imply its endorsement, recommendation, or favoring by the United States Government or any agency thereof or its contractors or subcontractors. The views and opinions of authors expressed herein do not necessarily state or reflect those of the United States Government or any agency thereof. 


\title{
New figuring model based on surface slope profile for grazing incidence reflective optics
}

Authors

\author{
Lin Zhou ${ }^{\text {abc }}$, Lei Huang ${ }^{b}$, Nathalie Bouet ${ }^{\text {b }}$, Konstantine Kaznatcheev ${ }^{b}$, Matthew Vescovi ${ }^{b}$, \\ Yifan Dai ${ }^{\mathrm{ac}}$, Shengyi $\mathrm{Li}^{\mathrm{ac}}$ and Mourad Idir ${ }^{\mathrm{b} *}$
}

${ }^{\mathrm{a} C o l l e g e ~ o f ~ M e c h a t r o n i c ~ E n g i n e e r i n g ~ a n d ~ A u t o m a t i o n, ~ N a t i o n a l ~ U n i v e r s i t y ~ o f ~ D e f e n s e ~ T e c h n o l o g y, ~} 109$ Deya Rd, Changsha, Hunan, 410073, People's Republic of China

${ }^{b}$ NSLS II, Brookhaven National Laboratory, PO box 5000, Upton, New York, 11973, USA

' Hu’nan Key Laboratory of Ultra-precision Machining Technology, 47 Yanzheng St, Changsha, Hunan, 410073, People's Republic of China

Correspondence email: midir@bnl.gov

Surface slope profile is widely used in the metrology of grazing incidence reflective optics instead of surface height profile. Nevertheless, the theoretical and experimental model currently used in deterministic optical figuring processes is based on surface height, not on surface slope. This means that the raw slope profile data from metrology need to be converted to height profile to perform the current height-based figuring processes. The inevitable measurement noise in the raw slope data will introduce significant cumulative error in the resultant height profiles. As a consequence, this conversion will degrade the determinism of the figuring processes, and will have an impact on the ultimate surface figuring results. To overcome this problem, we proposed an innovative figuring model, which directly uses the raw slope profile data instead of the usual height data as input for deterministic process. In this paper, first the influence of the measurement noise on the resultant height profile is analyzed, and then a new model is presented, finally a demonstration experiment was carried out using a one-dimensional ion beam figuring process to demonstrate the validity of our approach.

Keywords: ion beam figuring; synchrotron optics; one-dimensional; surface slope 


\section{Introduction}

Surface slope is widely used in the metrology of grazing incidence reflective optics instead of surface height (Idir et al., 2014; Qian et al., 2015; Siewert et al., 2004; Siewert et al., 2012; Siewert et al., 2014). The wide use of surface slope benefits from the two following factors. (1) For most grazing incidence reflective optics, a slope profile can better specify the optical performances than a height profile (Danjon, et al., 1990). (2) A slope profile is relatively easier to measure than height profile for most grazing incidence reflective optics. Slope profilers are more "universal” test system and do not require null configuration. Interferometers are limited in aperture and grazing incidence reflective optics with long size, or with steep curvature, are hard to be measured with classical interferometers. Therefore, most grazing incidence reflective optics are measured by slope measuring systems, such as Long Trace Profiler (LTP) (Qian et al., 2007; Thomasset et al., 2005), Nanometer Optical Machine (NOM) (Alcock et al., 2010; Siewert et al., 2014), or 2D Stitching Shack Hartmann Optical Head (SSH-OH) (Idir et al., 2014). The output data of all these slope measuring systems are $1 \mathrm{D}$ slope profiles or $2 \mathrm{D}$ slope maps.

However, the current figuring model used in the deterministic fabrication is based on the surface height profile. We called it height-based figuring (HF) model. In the HF model, the height amount removed by a figuring process is the convolution of the height removal function and the dwell time of the process (Drueding et al., 1995). To perform a figuring process with slope measuring systems, the original output, raw slope profile data, need to be converted by integration into height profile. Because the raw slope profile data inevitably contain measurement noise, although the noise can be very low in the slope domain, it will introduce a significant accumulation error in the resultant height profile (Artemiev et al., 2012). We will illustrate this issue in the following section. The introduced accumulation error will degrade the determinism of the figuring processes and will have an inevitable impact on the ultimate surface figuring results. The conversion of slope data to height data will always cause a shift of the positioning of the topography with respect to the sample coordinates.

In our previous works (Zhou et al., 2016), using the traditional HF mode and a slope measuring system (Idir et al., 2014), we had achieved correction accuracies on grazing incidence reflective optics below 1nm RMS. But it was very difficult to reach below 0.5nm RMS, although both the IBF process and the slope metrology have excellent repeatability. We notice that the 
introduced accumulation errors from the conversion of slope to height may cause the problem and limit further accuracy. Peverini et al. in their work to profile aspherical X-ray mirrors with a stationary broad ion beam and two movable blades, used slope profiles instead of height profiles to calculate the moving speed of the blades, and got better results (Peverini et al., 2010). However, their method and discussion are only limited to their particular case. In our study, we consider this problem in a more general way and developed a new figuring model, which uses the raw slope profile data instead of the converted height profile data. In this new slope-based figuring (SF) model, because the process calculations use directly the raw slope data, the accumulation errors from the conversion of slope to height can be avoided, and high convergence rate and high accuracy can be more easily achieved.

In the following section of this paper, the accumulation error introduced by the conversion from slope to height is analyzed, then the new SF model is presented, and a demonstration experiment was carried out using a one-dimensional (1D) ion beam figuring (IBF) process to demonstrate the validity of our approach.

\section{Error analysis}

If we have a set of slope measurement data $s_{1}, s_{2}, s_{3}, \cdots, s_{n}$, for positions $x_{1}, x_{2}, x_{3}, \cdots, x_{n}$ with an increment length $d$. The simplest way to convert the slope data into height data is to apply a Riemann summation

$$
h_{k}=d \sum_{i=1}^{k} s_{i} .
$$

However, in any measurement, the measuring error is inevitable. In this case, if the slope measurement at position $x_{i}$ has an error $\varepsilon_{i}$, then the corresponding height error at position $x_{k}$ is

$$
e_{k}=d \sum_{i=1}^{k} \varepsilon_{i} .
$$

If we assume the slope errors to be random, independent and identically distributed, with zero

mean and variance $\sigma^{2}$, i.e. $\varepsilon_{i} \sim N\left(0, \sigma^{2}\right)$, according to the random theory, the resultant height error at position $x_{k}$ is subject to a k-dimensional Gaussian distribution

$$
e_{k} \sim N\left(0, k d^{2} \sigma^{2}\right) .
$$


This means that in the converted height data, the uncertainty at the $k^{\text {th }}$ point will be enlarged $k$ times compared to the slope data. Artemiev (Artemiev et al., 2012) has noted that the trapezoid rule, Simpson's rule and other Newton-Cotesapproximate integration techniques suffer essentially the same drawback as the Riemann summation, even the advanced discrete Fourier transform (DFT) method (Campos et al., 2002), the uncertainty at the height data are significantly enlarged as well.

Here, we illustrate the influence of the noise in the slope profile data on the converted height profiles by MATLAB ${ }^{\circledR}$ simulation. Considering a $250 \mathrm{~mm}$ long mirror, with $1.2 \mathrm{~mm}$ measuring step, and a noise distribution variance of 10nrad, two noise sequences (slope profile errors, in units of $\mu \mathrm{rad}$ ) generated by MATLAB ${ }^{\circledR}$ command “ $0.01{ }^{*}$ randn $(1,208)$ " are shown in Figure 1a and $1 \mathrm{~b}$ in solid blue lines. According to the Eq. (2), the cumulative sum of each sequence, multiplied by a factor of 1.2 (measuring step, $\mathrm{mm}$ ), can be derived to the height profile errors (in $\mathrm{nm})$, which are shown in Figure 1 in green dotted lines.
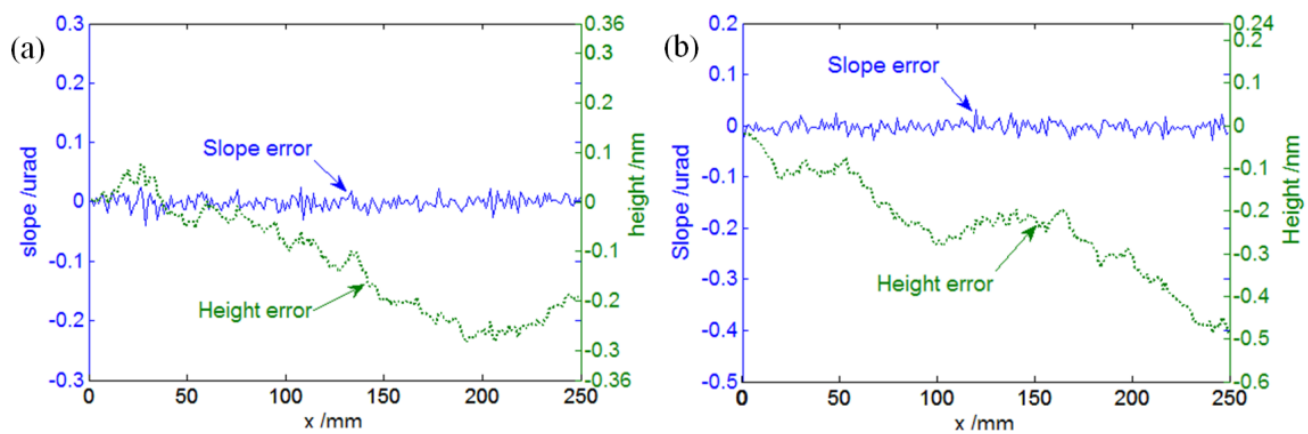

Figure 1 Two examples illustrating the influence of the slope noises on converted height profile.

The simulation results show that although the measurement noises are as small as 10nrad, the converted height error are in the level of $0.2 \sim 0.3 \mathrm{~nm}$ for a $250 \mathrm{~mm}$ length, and can be sometimes even greater than $0.4 \mathrm{~nm}$. These errors are too big to be neglected in the testing and figuring of $\mathrm{X}$ ray diffraction limited mirrors, where the acceptable height errors must be below $(\lambda / 27 \theta)$ rms $(\sim$ $0.6 \mathrm{~nm} \mathrm{rms}$, for $\lambda=0.05 \mathrm{~nm}$ and $\theta=3 \mathrm{mrad}$ ) (Pardini et al., 2015). Since all the calculations in the conversion from slope to height are linear, if the measurement noise in the slope profile increases, the converted height error will be linearly increased. Furthermore, with the increase of the length of the mirror, the number of the measurement points will be increased, and the converted height error will be linearly increased as well. 
In brief, although the measurement noises in slope profile data are very small in slope domain, the introduced error in height domain may be significant enough to affect the determinism of figuring ultra-precision X-ray diffraction limited mirrors.

\section{Slope-based figuring model}

In the traditional HF model, the height amount removed by a figuring process is the convolution of the height removal function and the dwell time of the process. Here, we only discuss the onedimensional case

$$
\begin{aligned}
r(x) & =\int_{-\infty}^{+\infty} p(x-u) \tau(u) d u \\
& =p(x) \otimes \tau(x),
\end{aligned}
$$

where, $r(x)$ is the height amount removed by the process, $p(x)$ is the height removal function of the process, $\tau(x)$ is the dwell time in the process, and $\otimes$ stands for the convolution operation. By differentiating Eq. (4),

$$
\begin{aligned}
\frac{d r(x)}{d x} & =\frac{d \int_{-\infty}^{+\infty} p(x-u) \tau(u) d u}{d x} \\
& =\frac{\int_{-\infty}^{+\infty} d p(x-u) \tau(u) d u}{d x}
\end{aligned}
$$

We get

$$
\begin{aligned}
s(x) & =\int_{-\infty}^{+\infty} q(x-u) \tau(u) d u \\
& =q(x) \otimes \tau(x),
\end{aligned}
$$

where, $s(x)=d r(x) / d x$ is the derivative of the height $r(x)$, i.e., the slope; $q(x)=d p(x) / d x$ is the derivative of the height removal function, which we refer to as the slope removal function.

Equation (6) is the model used to describe a deterministic optical figuring process based on slope profile. It reveals that the slope amount removed by a figuring process is the convolution of the slope removal function and the dwell time in the process. This general formula can be used in other applications where slope profiles are more interested than height.

According to Eq. (6), a deconvolution process is necessary to solve the dwell time $\tau(x)$ from the slope profile error $s(x)$ and the slope removal function $q(x)$. This calculation is the same as using Eq. (4) to solve the dwell time. Therefore, most of the deconvolution algorithms (Carnal et 
al., 1992; Drueding et al., 1995; Zhou et al., 2007; Liao et al., 2014) applied in the HF model can be used in the new SF model.

With this new SF method, the slope profile data doesn't have to be converted to height profile data. Therefore, the introduced accumulation error from the conversion can be avoided and the determinism of the figuring processes can be improved.

\section{Experimental results and discussion}

Experiments were carried out on a 1D IBF system developed at BNL (Zhou et al., 2016). Figure 2a shows the obtained height removal function. By differentiation, we can get the slope removal function, as shown in Figure 2b.
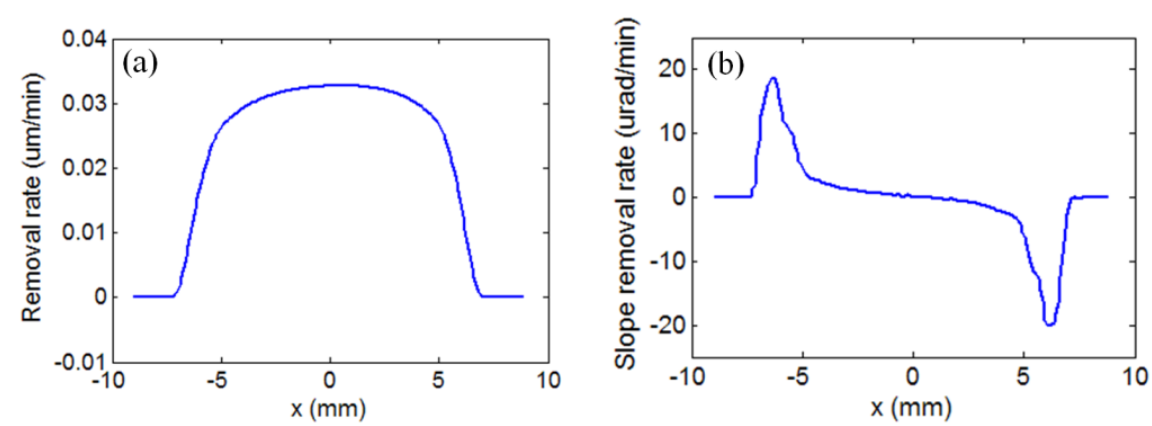

Figure 2 The removal functions in 1D IBF process. (a) Height removal function. (b) Slope removal function.

The shape of the slope removal function appears to be "strange" compared to the traditional height removal function, but this is not an issue for a deterministic optical figuring process. The only mandatory property for a removal function of a deterministic figuring process is stability. The matrix-based algorithm (Carnal et al., 1992; Zhou et al., 2007) can deal with this "strange” removal function. We use this algorithm to solve the dwell time in our 1D IBF process.

The parameters in our figuring demonstration experiment are the same as those in the experiment to obtain the removal function. The sample in this demonstration is a plane silicon mirror. After 3.3 minutes of 1D IBF process, the surface slope profile error on the central line of the sample was reduced from $0.40 \mu \mathrm{rad}$ RMS to $0.15 \mu$ rad RMS (see Figure 3a). The surface slope was measured by a slope measuring instrument developed at BNL (Idir et al., 2014). The corresponding height profile error was reduced by a factor of 10 from $3.84 \mathrm{~nm}$ RMS to $0.38 \mathrm{~nm}$ RMS (see Figure 3b). This experimental result shows that the deterministic figuring process 
driven by slope metrology data is very successful. Here, the height profile errors shown in Figure $3 \mathrm{~b}$ are only used to illustrate how the surface profile is corrected, but those height profiles are neither calculated nor used in our figuring process.
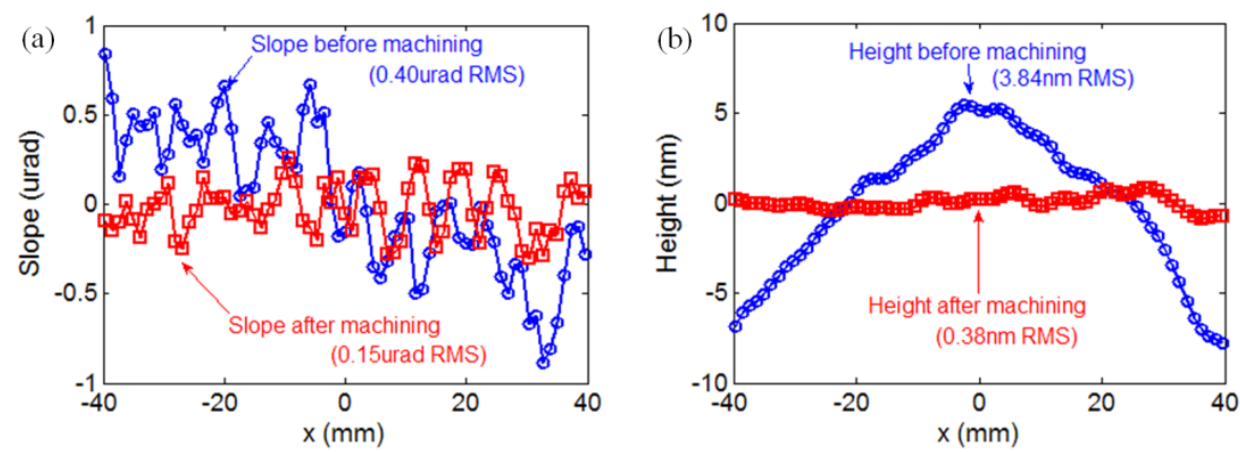

Figure 3 The experimental result of 1D IBF using the SF method. (a) Slope profile error. (b) Height profile error.

\section{Conclusion}

Due to the influence of measuring noise, the resulted height profile converted from raw slope data is imprecise. Subject to a Wiener random sequence, the error uncertainty at the $k^{\text {th }}$ point at the converted height profile will expand $k$ times than that of the raw slope profile. The traditional HF model based on the converted height profile is therefore not suitable to the processes with raw slope data from slope measuring system. We proposed a SF model, which uses the raw slope data instead of converted height data to perform deterministic figuring. The SF model reveals that, in a deterministic optical figuring process, the slope amount removed by afiguring process is the convolution of the slope removal function and the dwell time in the process, where, the slope removal function is the derivative of the height removal function. With this new SF model, the dwell time used to control the figuring process can be directly solved from the raw slope data, and the error-yield conversion from slope to height can be avoided. Therefore, a figuring process with SF model can improve the determinism of the process and achieve a better result.

Acknowledgements This work was supported by the US Department of Energy, Office of Science, Office of Basic Energy sciences, under contract No.DE-AC-02-98CH10886. Lin Zhou was supported by the National Natural Science Foundation of China (No. 91323302) and the Program for New Century Excellent Talents in University (No. NCET-13-0165). The authors acknowledge Ray Conley for his support during the beginning of this project. 


\section{References}

Alcock, S., Sawhney, K., Scott, S., Pedersen, U., Walton, R. \& Siewert, F. (2010). Nucl. Instrum. Meth. A. 616, 224-228.

Artemiev, N., Merthe, D., Cocco, D., Kelez, N., McCarville, T., Pivovaroff, M., Rich, D., Turner, J., McKinney, W. \& Yashchuk, V. (2012). Proc. SPIE. 8501, 2195-2197.

Campos, J., Yaroslavsky, L. P., Moreno, A. \& Yzuel, M. J. (2002). Opt. Lett. 27(22), 1986-1988.

Carnal, C., Egert, C. \& Hylto, K. (1992). Proc. SPIE. 1752, 54-62.

Danjon, A. \& Couder, A. (1990). Lunettes et télescopes. Blanchard, réédition.

Drueding, T., Bifano, T. \& Fawcett, S. (1995). Precis. Eng.17, 10-21.

Idir, M., Kaznatcheev, K., Dovillaire, G., Legrand, J. \& Rungsawang, R. (2014). Opt. Express. 22, 27702781.

Idir, M., Huang, L., Bouet, N., Kaznatcheev, K., Vescovi, M., Lauer, K., Conley, R., Rennie, K., Kahn, J., Nethery, R. \& Zhou, L. (2015). Rev. Sci. Instrum.86,

Liao, W., Dai, Y., Xie,X. \& Zhou, L. (2014), Appl. Opt. 53, 4266-4274.

Pardini, T., Cocco, D. \& Hau-Riede, S. P. (2015). Opt Express. 23, 31889-31895.

Peverini, L., Kozhevnikov, I.V., Rommeveaux, A., Vaerenbergh, P.V., Claustre, L., Guillet, S., Massonnat, J.-Y., Ziegler, E. \& Susini, J. (2010). Nucl. Instrum. Meth. A. 616, 115-118.

Qian, S. \& Takacs, P. (2007). Opt. Eng. 46, 043602.

Qian, S., Geckeler, R., Just, A., Idir, M. \& Wu, X. (2015). Nucl. Instrum. Meth. A. 785, 206-212.

Siewert, F., Noll, T., Schlegel, T., Zeschke, T. \& Lammert,H. (2004). Proc. AIP 705, 847-850.

Siewert, F., Buchheim, J., Boutet, S., Williams, G., Montanez, P., Krzywinski, J. \& Signorato, R. (2012). Opt Express. 20(4), 4525-36.

Siewert, F., Buchheim, J., Zeschke, T., Störmer, M., Falkenberg, G. \& Sankari, R. (2014). J. Synchrotron Rad. 21, 968-975.

Thomasset, M., Brochet, S. \& Polack, F. (2005). Proc. SPIE. 5921, 592102.

Zhou, L., Dai, Y., Xie, X., Jiao, C. \& Li, S.(2007). Nanotechnol.Precis. Eng.5, 107-112.

Zhou, L., Idir, M., Bouet, N., Kaznatcheev, K., Huang, L., Vescovi, M., Dai, Y. \& Li, S. (2016). J. Synchrotron Rad.23, 182-186. 